\title{
Moments and Points in an Interval-Based Temporal Logic
}

\author{
James F. Allen and Patrick J. Hayes* \\ Departments of Computer Science and Philosophy \\ The University of Rochester, Rochester, NY 14627
}

TR 180

December 1987

\begin{abstract}
The literature on the nature and representation of time is full of disputes and contradictory theories. This is surprising since the nature of time does not cause any worry for people in their everyday coping with the world. What this suggests is that there is some form of common sense knowledge about time that is rich enough to enable people to deal with the world, and that is universal enough to enable cooperation and communication between people. In this paper, we propose such a theory and defend it in two different ways. We axiomatize a theory of time in terms of intervals and the single relation MEET. We then show that this axiomatization subsumes Allen's interval-based theory. We then extend the theory by formally defining the beginnings and endings of intervals and show that these have properties we normally would associate with points. We distinguish between these point-like objects and the concept of moment as hypothesized in discrete time models. Finally, we examine the theory in terms of each of several different models.
\end{abstract}

This research was supported in part by the National Science Foundation under Grant DCR-8502481 and the Air Force Systems Command, Rome Air Development Center and the Air Force Office of Scientific Research under Contract F30602-85-C0008, which supports the Northeast Artificial Intelligence Consortium (NAIC).

*P.J. Hayes's current address is Xerox Palo Alto Research Center, 3333 Coyote Hill Road, Palo Alto, CA, 94304. 


\section{Introduction}

The literature on the nature and representation of time is full of disputes and contradictory theories. This is surprising since the nature of time does not cause any worry for people in their everyday coping with the world. What this suggests is that there is some form of common-sense knowledge about time that is rich enough to enable people to deal with the world and universal enough to enable cooperation and communication between people. In this paper we propose such a theory and defend it in two different ways.

First, the theory is powerful enough to include the distinction between intervals (i.e., times corresponding to events with duration) and moments (i.e., times corresponding to instantaneous events), as well as allowing substantial reasoning about temporal ordering relations (including the abilities described in [Allen, 1984]). In addition, it includes a formalization of the beginnings and endings of events by introducing the corresponding beginnings ind endings of times. We show that beginnings and endings act in many ways like moments, yet can be distinguished from them.

Second, the theory has as allowable models a number of the temporal models that are suggested in the literature. This includes models that equate time with intervals and points on the real number line, models that hypothesize discrete time, and any model that mixes real points and intervals. Our claim is that if our common-sense theory of time excluded any one of these models, then there would be no debate as to whether that model was valid, since in that case our own primitive intuitions on the matter would be extremely clear.

An important intuition that guides us is that time is only a projection of events that occur and properties that hold. If the universe did not change, there would be no time. Any sort of event or happening that can be described or thought of has a corresponding time, and the universe of times consists of these. It is important to note, however, that we are not constructing a logic of events such as that described in Kamp [1979]. In an event calculus, two events may be temporally identical yet distinct. In the interval logic, two identical intervals are by necessity equal.

A consequence of this view is that we will start with a neutral term, time period, and introduce properties of such periods as they are needed. The primary requirement is that time periods must be able to meet, which occurs when two periods neither share any time in common nor have any time between them. For example, if some property $P$ changes from being true to being false, the period where $P$ is true meets the period where $P$ is false. These two periods neither overlap (in which case $P$ would be simultaneously true and false!) nor are separated by any time (in which case there would be a truth gap). The importance of the meets relation in naive theories of time has been argued elsewhere (e.g., [Allen, 1984]) and will not be defended further here. 
Let us consider the issue of how small a time period might be or, in fact, whether they must have a duration at all. There seem to be several situations that intuitively require small periods. Below we introduce two that will be examined at length in this paper.

A moment is simply a nondecomposable period. Given the above discussion, it should be clear that nothing could change during a moment. If there were an observable change during the moment, then we could subdivide it in to the part before the change and the part after. A natural example of a moment is the time taken for a flash of lightning, or a flash of a strobe light, where the world appears to be frozen. Another example is the time it takes to take a picture. As one can see, what might be considered a moment may depend very much on the context, and on the measuring instruments available. A moment in everyday life may be a bewildering complex interaction of events to a researcher in particle physics!

A point, in contrast to a moment, has a zero duration time and arises in situations where we need to reason about the beginnings and endings of events. The beginning of a race is the time of transition from the race not being in progress to the race being in progress. Such points cannot be viewed as having duration unless we are willing to accept truth gaps (i.e., is the race on or not yet begun at the beginning of the race?). A classic example, from elementary physics, concerns the path of a ball thrown vertically into the air. We all learned that there is a time when the ball is stationary, and is neither rising nor falling. Depending on how literally we take this fact, the time the ball is stationary will be a moment or a point. One version is that the ball is stationary only for a time of zero duration, which in fact is the point where the interval in which the ball is rising meets the interval in which it is falling. If, on the other hand, you believe that the ball is stationary for some non-zero duration, then the time is a moment. The interval in which the ball is rising meets the moment where it is stationary, which in turn meets the interval where it is falling. In this case, the rising interval is strictly before the falling interval.

In Section I, we axiomatize a theory of time in terms of the single relation meets. It is then shown in Section $I I$ that this axiomatization subsumes the interval-based theory proposed in [Allen, 1983; 1984]. We then extend the theory to point-like time periods. Section III discusses the distinction between moments and points, and Section IV formally defines the beginnings and endings of intervals and shows that they have the properties we normally would associate with points. Section V gives a set-theoretic definition of points providing an alternative analysis to that in Section IV. Section VI discusses the properties of moments, and Section VII examines the theory in terms of each of several different models. 
The proofs of the theorems are not included in this paper. Most of them are fairly straightforward. Where this is not the case, we try to give an intuitive argument of why it is true. All the proofs are included in the appendix.

We will be using the first-order predicate calculus with equality throughout, with the operators \& (and), $\vee$ (or), $\oplus$ (exclusive-or), つ (implication), $\equiv$ (equivalence), and the standard quantifiers $\forall$ (universal) and $\exists$ (existential). Scoping will be indicated by parentheses or by the dot notation. For example:

$$
\forall i, j . P(i, j) \supset Q(i, j)
$$

is the same as

$$
\forall i, j(P(i, j) \supset Q(i, j))
$$

\section{An Axiomatization of Interval Time}

We start the formal development by positing a non-empty class of objects in our ontology that we shall call time periods. These are intended to correspond to our intuitive notion of when some event occurs. We do not, at this early stage, make any commitment as to whether all times are decomposable or not.

We define the single relation meets between times and formulate its properties. We deliberately make no commitments as to the structure of the time periods--whether they are continuous, divisible, etc. The single intuitive idea we try to capture is that periods are all contained in a single "time-line." We will write the meets relation as a colon in formal expressions, and use chained infix notation to compactly express a conjunction of meetings, so that "i meets $\mathrm{j}$ " is written as $i: j$, and " $\mathrm{i}$ meets $\mathrm{j}$ and $\mathrm{j}$ meets $\mathrm{k}$ and $\mathrm{k}$ meets $\mathrm{l}$ " will be written $i: j: k: l$.

The first axiom is based on the intuition that the "place" where two periods meet is unique and closely associated with the periods. Thus if two periods both meet a third, then any period met by one must also be met by the other. This can be compactly stated as:

$$
\forall i, j, k, l .(i: j \& i: k \& l: j) \supset l: k
$$

Next, we need to ensure that meeting-places are totally ordered. This axiom uses a technique for asserting that one meeting-place is earlier than another that we will make extensive use of later. This says that if period $i$ meets $j$ and period $k$ meets $l$, then exactly one of the following holds:

1) $i$ meets $l$;

2) there is an $m$ such that $i$ meets $m$ and $m$ meets $l$;

3) there is an $n$ such that $k$ meets $n$ and $n$ meets $j$. 
In other words, we have exactly three possible cases, shown in Figure 1, for any four times $i, j, k$, and $l$.

$$
\begin{aligned}
& \begin{array}{l|l|l|}
-i-\mid-j-1 & -i-\mid-j- & \cdots i-\mid-j- \\
-k-\mid-l-1 & |-m-| & |-n-|
\end{array}
\end{aligned}
$$

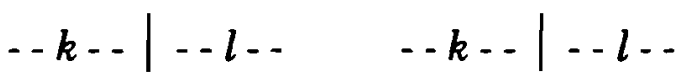

\section{$\begin{array}{lll}\text { Case } 1 & \text { Case } 2 & \text { Case } 3\end{array}$}

Figure 1: The Three Possible Orderings of $i, j, k$, and $l$ in Axiom M2

(M2)

$$
\forall i, j, k, l .(i: j \& k: l) \supset i: l \oplus(\exists m . i: m: l) \oplus(\exists m . k: m: j)
$$

Notice that if $i: l$ then $k \cdot j$, by M1. The exclusive-ors in this axiom have some quite powerful consequences. In particular, they ensure that there can be no circular times. Suppose $i$ meets itself: then the appropriate instance of M2 allows us to conclude both that $i: i$ and that there exists an $m$ with $i: m: i$ (since $i: i: i$ ). Then two cases of the conclusion of M2 would be simultaneously true, contradicting the exclusivity of the exclusive-or. Thus we have the lemma:

(ML1) $\quad \forall i . \neg i: i$

Similarly, assume $i: j$ and $j: i$. In this case axiom M2 allows us to conclude both $\exists m . i: m: i$ and $\exists m . j: m: j$, again violating the exclusivity of the exclusive-or. Thus we have:

$$
\forall i, j \cdot i: j \supset \neg(j: i)
$$

More generally, the above argument shows that there is no period $m$ such that $i: m: i$, i.e.,

$$
\forall i . \neg \exists m . i: m: i
$$

Next we need some existence axioms. The simplest merely does not allow time to start or stop.

$$
\forall i \exists j, k \cdot j: i: k
$$

We need one further existence axiom. The presence of a period between any identifiable meeting places is a very basic fact in this world-picture: it is the presence or otherwise of such intervals that "encodes" time-order, for example, as in M2. If two meeting-places are separated by a sequence of intervals, we 
need to be able to conclude that this sequence itself constitutes a longer period. Here, for the first time, periods exist which have an internal structure.

$$
\forall i, j . i: j \supset(\exists k, m, n . m: i: j: n \& m: k: n)
$$

Notice the use of auxiliary times ( $m$ and $n$ ) whose function is merely to ensure that $k$ and $i$ start at the same point and $k$ and $j$ finish at the same point. This is another trick which we will use again. That such times exist on either side of $i$ and $j$ is of course guaranteed by M3.

Our final axiom in the basic theory of meeting is a simple extensionality assumption which says that there is only one time period between any two meeting-places. Without this assumption, time could branch arbitrarily in the past or future directions.

$$
\forall i, j, k, l .(i: j: l \& i: k: l) \equiv j=k
$$

It now follows that the period whose existence is guaranteed by M4 must be unique, since if $m: i: j: n$ and $m: k: n$ and $m: k^{\prime}: n$, then $k=k^{\prime}$. Thus we can think of there being a function from two adjacent periods to this longer period, which we will call the ordered union or sum of $i$ and $j$, and write as $i+j$, so that M4 can be restated as:

$$
\forall i, j . i \cdot j \supset(\exists m, n . m: i \cdot j: n \& m:(i+j): n)
$$

It is easy to show that + is associative, so we will let ourselves abuse notation in the future by writing expressions such as $i+j+k$.

This is the complete basic set of axioms concerning the meets relation. These are enough to reconstruct the larger theory of interval relations. Simple definitions of the complete set of possible relationships between intervals can be given in terms of meets. The definitions work by hypothesizing intervals which represent the gaps between the ends of the given intervals, as in axiom M2, and using auxiliary intervals to tie loose ends together, as in M4.

For example, we can define a relationship BEFORE to hold between two times only if there is a period which spans some time between them. Thus

$$
i B E F O R E j \equiv \exists k . i: k: j
$$

The relation STARTS, which holds between a time and one which is a continuation of it, can be defined by asserting the existence of an interval which, when added to the second, makes the first:

$$
i \operatorname{STARTS} j \equiv \exists k \cdot j=i+k
$$

If one expands the definition of + using M4.1, this becomes 
$i \operatorname{STARTS} j \equiv \exists k, m, n . m: i: k: n \& m: j: n$

All the definitions using + below are equivalent to formulae of this simple form, in which a number of times are asserted to exist that meet one another. The most complex is OVERLAPS, which requires five extra periods to be hypothesized.

\begin{tabular}{|c|c|c|}
\hline $\begin{array}{l}\text { Relation } \\
\text { Between I \& J }\end{array}$ & Definition & Inverse Relation \\
\hline BEFORE, b & $\exists k . I: k: J$ & AFTER, a \\
\hline OVERLAPS, o & $\begin{array}{l}\exists k, l, m . I=k+l \& J \\
\quad=l+m\end{array}$ & OVERLAPPED-BY, oi \\
\hline STARTS, s & $\exists \mathrm{k} . J=I+k$ & STARTED-BY, si \\
\hline FINISHES, f & $\exists k \cdot J=k+I$ & FINISHED-BY, fi \\
\hline DURING, d & $\exists k, l . J=k+I+l$ & CONTAINS, di \\
\hline MEETS, : & $I: J$ & MET-BY, mi \\
\hline
\end{tabular}

Figure 2: The Six Relationships Between $I$ and $J$

Six relationships between periods are defined in Figure 2, which also gives the shorter notation which we will use in formulae and the names of the inverses. We include the meets relation above so that we have a convenient notation for the inverse of meets (i.e., $m i$ ) in later discussion. We will also use the abbreviations for disjunctions used in [Allen, 1983]. Thus $j$ (oois $f d$ ) $i$ is shorthand for the formula

$$
\begin{aligned}
& \text { jOVERLAPS } i \vee j O V E R L A P P E D-B Y i \vee j S T A R T S i \vee \\
& j \text { FINISHES } i \vee j D U R I N G i
\end{aligned}
$$

The thirteenth relationship is equality. If we take equality as a nonlogical symbol, then axiom M5 can be taken as its definition in the same style as the others shown.

In addition, we will define a relation IN to include all the relationships involving "containment," i.e.,

$$
i I N j \equiv i \text { DURING } j \vee i \text { STARTS } j \vee i \text { FINISHES } j \vee i=j
$$

An intersection operator on times also proves useful throughout in the proofs. Let $I ! J$ be the intersection of $I$ and $J$, which is defined as follows: $I ! J$ is the interval that exists if there exists a subinterval common to both $I$ and $J$, 


$$
\exists i .(i I N I) \&(i I N J) \supset(I ! J I N I) \&(I ! J I N J)
$$

and is the largest common subinterval,

$$
\forall i \text {. (iINI) \& (iINJ) つ iINI!J }
$$

We can prove that $I ! J$ is unique if it exists (Lemma IL1), and it exists whenever $I$ and $J$ overlap in the intuitive sense of the word, i.e., I (o oi s si f fi $d d i=$ ) $J$ (Lemma IL2). Since this is all derivable from axioms M1-M5, axioms I1 and I2 can be regarded as a definition of this notation.

Some other lemmas will be useful later. In particular,

Lemma IL3: If $i=a+b$ and $j: b$, then $i ! j$ exists and is equal to $a ! j$. If $i=a+b$ and $a: j$, then $i ! j$ exists and is equal to $b ! j$.

\section{Capturing the Behavior of an Interval-Based Temporal Reasoner}

The question now arises as to whether the above axiomatization of meet and the definitions of the other relationships totally capture the behavior of the interval logic in [Allen, 1983]. This turns out to be the case, although it is tedious to show. Before we expand on this, the intended meaning of the transitivity table in [Allen, 1983] should be made clear. Consider an entry for the relations MEETS and BEFORE, which has the value BEFORE. If encoded in FOPC, this entry would have the following interpretation:

$$
\forall a, b, c . a \text { MEETS } b \& b B E F O R E c \supset a B E F O R E c
$$

In particular, the meaning of the table entries cannot be defined by a biconditional (i.e., iff) statement. In fact, strengthening $\left({ }^{*}\right)$ to a biconditional would actually force all intervals to be decomposable (among other things), a characteristic that we explicitly do not want in the basic model.

We can prove that, for any two intervals $I$ and $J$, then exactly one of the thirteen interval relationships possible holds between them. We can also show that the transitivity table in [Allen, 1983] is a result of the above axiomatization. This had to be shown entry by entry through the table, following the intuitive reasoning by possible cases which was used to construct the table originally. The proof, while long, is simple, as it only involves the repeated application of the ordering axiom M2. For example, given $I, J$, and $K$ such that IOVERLAPS $J$ \& J DURING K, we know

$$
\begin{array}{r}
\exists a, b, c, d, e . a: I: d: e \& \\
a: b: J: c \& \\
b: c: d
\end{array}
$$




$$
\begin{gathered}
\exists f, g, h, j \cdot f: g: J: h j \& \\
f: K: j
\end{gathered}
$$

These facts can be presented pictorially as in Figure 3 .

$$
\begin{aligned}
& --a--|-b--|--c-.|--d--|-e-- \\
& |-\ldots . . . . . .| \\
& |-\ldots \ldots \ldots|
\end{aligned}
$$

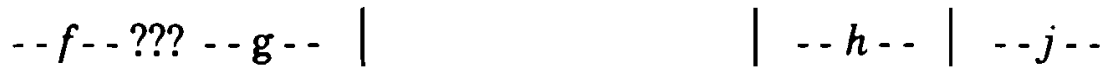

$$
\begin{aligned}
& \text { ??? - } \ldots \ldots \ldots \ldots
\end{aligned}
$$

Figure 3: IOVERLAPS J \& J DURING K

Using Axiom M3, and the facts $a: b \& f: g$, we have three cases:

Case 1: $\quad a$ meets $g$, and hence $b=g$ (since $a: b: J \quad \& a: g: J$ ); but then we have

$$
a: I: d+h: j \& a: K: j,
$$

which by definition entails I STARTS K

Case 2: $\exists m \cdot a: m: g$; in which case we have

$$
\begin{aligned}
& a: m: g+c: d+h: j \& \\
& a: I: d+h: j \& \\
& a: m: K: j
\end{aligned}
$$

which by definition entails IOVERLAPS K

Case 3: $\exists n \cdot f: n: b$; in which case we have

$$
f: n: I: h: j \& f: K: j
$$

which by definition entails I DURING K

Thus, we have the fact that

$$
\text { (IOVERLAPS J \& J DURING K) つI(sod) } K
$$


which is one of the entries in the transitivity table in [Allen, 1983]. Some additional derivations of entries are shown in the appendix.

This set of five axioms is of a manageable size for comparing different theories and for theoretical proofs. This is not to say, of course, that a reasoning system should be implemented solely in terms of the meets relation. There are important efficiency gains from using the larger set of primitives, as already described in [Allen, 1983].

\section{Time Moments and Time Points}

So far, times are all things with a duration, a "temporal extent," that are positioned next to one another. There is no explicit notion of a place or position in time, such as would be the denotation of a clock time. In this section we will introduce such time points in several different ways and show that they are all equivalent, which helps to bolster our intuition that the interval axioms introduced so far form a robust set of basic assumptions about time periods.

There are several intuitive accounts of what points are. The first takes them to be the "places" where one interval meets another, the times of transition. From this alone we can develop a precise formalization of points as the endpoints of intervals. The time that a ball thrown up in the air is at its peak, i.e., the time of transition from rising to falling, would be a point.

Other examples can be obtained by considering sentences describing the socalled accomplishment events, such as "I shot the gun." While such an event can be located in time (e.g., "I shot the gun at midnight"), it cannot be qualified by a duration (e.g., "I shot the gun for ten minutes") without invoking the notion of repeated occurrence. A single shooting of the gun cannot be described so. As expected, accomplishment sentences all involve transitions. In the above sentence, for example, the gun changes state from being loaded to being empty. The only cases where it appears that such an event can take time qualification actually are better analyzed as the time it took to get the event to occur. For example, we can say "It took ten minutes to shoot the gun," but here we are saying that it took ten minutes to do the actions that eventually caused the firing.

Note again the distinction between time points in this sense and very short, momentary, intervals which are the duration of extremely short events, but which do occupy time. For example, consider the flash with which an incandescent bulb burns out, plunging a room into darkness: here there are three intervals, each meeting the next, the middle one being the time occupied by the flashing event. Even though such moments seem instantaneous, they also intuitively still have a duration, and by this we can distinguish them from points. 
To elaborate, a sentence describing an event over a moment behaves the same way as a sentence describing a point event when duration modifiers are added. For example, "He flashed the light for three hours" must take a reading involving repetition. With moment events, however, if the duration modifier is small enough to satisfy general world knowlege, the modifier becomes acceptable for a single occurrence. For example, "He flashed the light for half a second" is perfectly acceptable as describing a single flash. For point events, no duration modifier, no matter how small, behaves in this way.

\section{Time Points}

We will introduce points as the "meeting places" of intervals, such as the point where the interval associated with "the ball rises" meets the interval associated with "the ball falls," or the point where the interval "the door is open" meets the interval "the door is closed." Points by definition have no duration, and we can only assert properties to hold at points in an indirect fashion by referring to an interval that contains the point in some way. For example, consider the example of the door closing while a light in the room stays on. The situation is as follows:

Let $O$ be the time door is open,

$C$ be the time door is closed, and

$L$ be the time light is on.

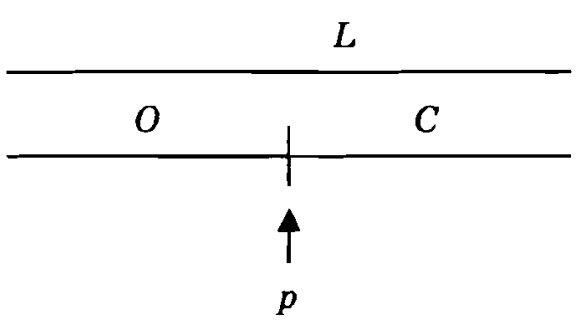

Let $p$ be the point where $O$ and $C$ meet. We can say that the light is on at $p$ since $p$ is contained in $L$. We cannot say either that the door is open at $p$ or closed at $p$, however, since neither $O$ nor $C$ contain $p$. Thus it may appear that there are truth gaps at time points (but not over intervals). In fact, we simply do not allow assertions at points in the normal sense.

In order to satisfy these intuitions, the set of points must be totally ordered, and there should be a 1:1 mapping between points and meetings of intervals that respects the point ordering, so that $p$ is before $q$ just when there is an interval with $p$ and $q$ corresponding to its endpoints. There are several ways to formalize this, and we will develop two here. The first uses two functions, BEGIN and END, from intervals to points; the second uses a trinary relation MEETS-AT between two intervals and a point. These are equivalent, given suitable fairly weak assumptions. 
From now on we will use the letters $p, q, r, s, \ldots$ as variables over points, continuing to use $i, j, k, \ldots$ as variables over intervals. This implicit typing convention could be replaced by annotating the axioms by suitable assumptions of the form INTERVAL(i) and POINT(p), where these are appropriate sortal predicates. We will ignore this complication in stating the axioms, but an automatic inference mechanism would of course need to be sensitive to the distinctions.

First, let us give for reference the bare axioms for a totally ordered set of points. We will not take these as part of our theory, but use them rather as a reference set of assumptions to be derived from the later constructions of points from intervals.

Irreflexive

$$
\forall p . \neg(p<p)
$$

Antisymmetric

$$
\forall p, q \cdot(\mathrm{p}<q) \supset \neg(q<p)
$$

Transitive

$$
\forall p, q, r .(p<q) \&(q<r) \supset(p<r)
$$

Furthermore, we want < to be a total ordering:

$$
\forall p, q .(p<q) \oplus(q<p) \oplus(p=q)
$$

The exclusive-ors in (P4) are not strictly necessary, or equivalently, (P1) is redundant. We include both for convenience and familiarity. In the final axiom, we ensure that the domain is not bounded with respect to " $<"$.

$$
\forall p \exists q, r . q<p<\mathbf{r}
$$

Let us introduce two functions on intervals that produce the endpoints. Thus for an interval I, begin(I) is the beginning point and end(I) is the ending point. We now can assert that the only points that exist are the places where two intervals meet:

$$
\forall p \exists i, j \cdot p=\operatorname{begin}(j) \& p=\operatorname{end}(i)
$$

Next, coincidence of endpoints corresponds to intervals meeting.

$$
\forall i, j . i: j \equiv(\operatorname{end}(i)=\operatorname{begin}(j))
$$


Notice the biconditional, which ensures that no points exist other than at places where intervals meet.

And finally the relation between meeting and point ordering is given quite compactly by the following, which again uses the idea that there must be an interval separating two times in order for one to be earlier than the other:

$$
\forall p, q \cdot p<q \equiv \exists i \cdot p=\operatorname{begin}(i) \& q=\operatorname{end}(i)
$$

This final axiom may be taken as a definition of $<$, and then it is fairly straightforward to derive P1-P5 from the interval axioms given earlier together with PBE1-PBE3. For example, the proof of transitivity follows from a couple of easy lemmas.

Lemma PL1: $\forall i, j . i: j \supset$ end $(j)=\operatorname{end}(i+j)$

Proof: From M4.1 it follows that there is an interval $k$ which both $j$ and $i+j$ meet: hence end $(j)=$ begin $(k)=e n d(i+j)$ by PBE2, used twice. QED.

Similarly: $\forall i, j . i: j \supset \operatorname{begin}(i)=\operatorname{begin}(i+j)$

Transitivity now easily follows, since if $p<q$ and $q<r$, then by PBE3 (used twice) there are intervals, say $i$ and $j$, with $p=\operatorname{begin}(i), r=e n d(j)$, and $i: j$. But then by M4.1 and the above lemmas, $p=\operatorname{begin}(i+j)$, and $r=e n d(i+j)$, hence by PBE3, $p<r$. QED.

The totality axiom $\mathrm{P} 4$ follows in a similar way, using the linearity axiom M2, for example. Another important lemma that follows from the above axioms is that any interval's beginning strictly precedes its ending, i.e.,

Lemma PL2: $\forall i . \operatorname{begin}(i)<\operatorname{end}(i)$

Full proofs for axioms P1 through P5 and lemma PL2 are given in the appendix.

An alternative approach using the MEETS-AT relation is also straightforward. This time, let us use a more compact notation. We will write $i ; p ; j$ to mean that the trinary relation MEETS-AT holds between the intervals $i$ and $j$ and the point $p$, rather than the more conventional but awkward MEETS$A T(i, j, p)$. The axioms corresponding to PBE1-PBE3 are now:

(PMA1) $\forall p \exists i, j . i ; p ; j$

(PMA2) $\forall i, j . i: j \equiv \exists p . i ; p ; j$

(In fact only half of PMA1 is strictly needed, e.g., $(\forall p . \exists i . i ; p)$, since the other half can then be derived. Without some assumption such as this, however, there could be "loose" points not associated with any intervals. If we wanted to allow 
such things, PMA1 could be omitted, but several later lemmas would then fail. In general, it would be difficult to establish any general properties of points, since many of them derive from their associations with intervals. It seems intuitive, in any case, that if a point can be identified, then intervals which end and begin at it should also be defined. A completely isolated point which is not the endpoint of any interval would seem to be an unintuitive idea.)

The definition of time ordering can now be given as

(PMA3) $\forall p, q \cdot p<q \equiv \exists i, j, k . i ; p ; j \& j ; q ; k$

Axiom PMA3 hypothesizes three intervals simply because it is a formal syntactic necessity: one cannot write just $p ; j$ or $j ; q$ in the language so far. This immediately suggests, however, an extension of the notation which does permit such expressions as ways of writing $p=\operatorname{begin}(j)$ and $q=\operatorname{end}(j)$, respectively. This notation directly displays the relations between an interval and its endpoints in much the same way as the infix notation for the MEETS relation did earlier. If the PBE axioms are rewritten in this notation, again allowing conjunctions to be compactly expressed as sequences, then PBE1 and PBE2 become identical respectively to PMA1 and PMA2, and PBE3 becomes

(PMA3.1) $\forall p, q . \exists i . p ; i ; q$

from which PMA3 is easily derived. We now also need a uniqueness axiom, which was previously implicit in the use of functional notation.

(PMA4) $\forall p, q, i .((i ; p \& i ; q)$ or $(p ; i \& q ; i)) \supset p=q$

We will use this semicolon notation to mean BEGIN, END, and MEETS-AT as needed by context.

To review, the notation so far allows expressions which have names of intervals separated by colons, and separated from names of points by semicolons. We can write, for example,

$$
i: j ; p ; k ; q ; l: m ; r
$$

as an abbreviation of

$i: j \& \operatorname{end}(j)=p \& \operatorname{begin}(k)=p \& \operatorname{end}(k)=q$

$\& \operatorname{begin}(l)=q \& l: m \&$ end $(m)=r$

Notice, however, that expressions of the forms $p: q$ or $i: p$ or $p: i$ are not well formed, since points cannot meet anything. All that an expression of the form $p: q$ could seem to be taken to mean would be $p=q$, since points are themselves meeting-places. However, as Van Benthem [1982] discusses, it is possible to construct mathematical structures which have such oddities as two-sided points. 
Several lemmas can be proven which establish intuitively sensible properties of points and relations between points and intervals. For example, in analogy to M5:

Lemma PL3: $\forall i, j, p, q .(p ; i ; q \& p ; j ; q) \supset i=j$

or alternately,

$$
\operatorname{begin}(i)=\operatorname{begin}(j) \& \operatorname{end}(i)=\operatorname{end}(j) \supset i=j
$$

Lemma PL4: $\forall i, j . i: j \supset(\forall p . i ; p \equiv p ; j)$

Lemma PL5: $\forall i . \exists p, q \cdot p ; i ; q$

Note that we cannot yet say that anything is true at a point, for the point is not yet the sort of entity at which things happen or are true. We can, however, claim indirectly that a proposition is true during a time which includes a point, as, for example, the light being on while the door is closing, for we can simply assert that it holds during an interval which overlaps the time the door is open. Less awkwardly, we will axiomatize the obvious notion of a point being in an interval.

(PMA5) $\forall p, i . p \in i \equiv \exists k, l . i=k+l \& k ; p ; l$

Note that by this definition, the endpoints of an interval are not in the interval.

\section{Set-Theoretic Derivations of Points}

So far, we have introduced points by simply hypothesizing their existence and adding axioms which describe them. The mathematical literature, however, contains several set-theoretic methods by which points may be explicitly constructed from intervals. The oldest of these, due in essentials to A.N. Whitehead, defines a point as a set of intervals which share a common intersection. (A famous alternative, due to Bolzano, defines a point as a set of pairs of intervals which bracket it from either side, forming successive approximations to the exact position. We will follow the former technique, but it can be shown that our interval axioms make them equivalent.) Our axioms M1M5, together with a small amount of elementary set theory, will support such a construction and allow its essential properties to be derived. We call such a set of overlapping intervals, a variant of the usual mathematical idea of a filter, a "nest."

In our case, since meeting of intervals is the basic relation, it is natural to group together all intervals which share or contain a meeting place. The beginning of an interval, for example, is defined to be the set of all intervals which intuitively touch or include the beginning in any way, and the end similarly. 


$$
\begin{aligned}
& { }^{*} \text { begin }(I)=\{j \mid \exists b, c \cdot b: I \& b: c \& \\
& j=b+c \vee j=c \vee j=b\}
\end{aligned}
$$

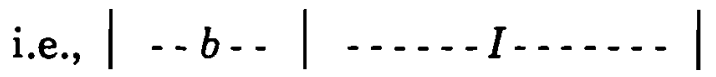

$$
\begin{aligned}
& \text { - c- - ? - _... ? - ? } \\
& \text { | } \\
& \text { or } \quad \mid--j-?_{-\ldots}-?^{-} \text {? } \\
& \text { or }|--j--| \\
& *_{\text {end }}(I)=\{\mathrm{j} \mid \exists a, b . I: b \& a: b \& \\
& j=a+b \vee j=a \vee j=b\}, \\
& \text { i.e., } \quad|\ldots \ldots \ldots-I \ldots \ldots| \ldots-\ldots \mid \\
& \text { ?- ? - - - ? - - a }
\end{aligned}
$$

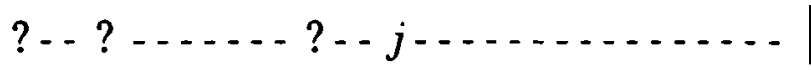

$$
\begin{aligned}
& \text { or } \quad ? \ldots ?-\ldots+\cdots,-j \ldots \\
& \text { or } \\
& |--j--|
\end{aligned}
$$

These sets, which are never empty in any model of the interval axioms, could also be defined using the 13-relation interval notation by:

$$
\begin{aligned}
& * \operatorname{begin}(i)=\{j \mid j(\text { o s:fidie si) } i\} \\
& *_{\text {end }}(i)=\{j \mid i(\text { of } i: f d \text { e } s) j\}
\end{aligned}
$$

For convenience, we can define a nest as a beginning or an ending:

$$
\operatorname{NEST}(p) \equiv \exists I \cdot p={ }^{*} \operatorname{begin}(I) \vee p={ }^{*} \text { end }(I)
$$

Suppose we have a model ${ }^{*} M M$ of the interval axioms M1 through M5. Then we will show that adding all the nests to the universe and interpreting the symbols "begin" and "end" respectively as *begin and *end results in a model for the larger set of axioms including PBE1 through PBE3. Thus our axiomatization is consistent with this mathematical construction of points from intervals.

We can define relations over the set of nests that gives them the properties of points. We shall say a nest $N$ is before a nest $M$ iff there is at least one interval in $N$ that is before some interval in $M$. For any two NESTS, $N$ and $M$

$$
N<M \equiv \exists n, m . n \text { is in } N \& m \text { is in } M \text { \& } n \text { BEFORE } m
$$


Now we can show some important properties about nests. First, nests are rather like filters. In particular, we can show that nests are closed under intersection.

Lemma NL1: $I$ is in *begin( $(I)$ and in *end(I)

The proof trivially follows from the definitions of *begin and *end and axiom M3.

Lemma NL2: If $i$ and $j$ are in a nest $N$, then either $i: j$ or $i ! j$ exists and is in $N$

The main result is that, given any two nests and the ordering relationships between nests defined above, the nests must either be equal or one is before the other. Furthermore, these possibilities are mutually exclusive.

Theorem N1: For any two nests $N$ and $M$, either $N<M, M<N$ or $N=M$

The proof of theorem $\mathrm{N} 1$ is given in the appendix. We can now show that nests have all the properties of points described in the last section by showing that the correlates of axioms PBE1 through PBE3 all hold for nests. Once this is done, all the other results in the last section fall out. In particular, we can now prove the following (where $p, q$ are now variables over nests):

(PL1') $\quad \forall i, j . i \cdot j \supset^{*}$ end $(j)={ }^{*}$ end $(i+j)$

$\forall i, j . i: j \supset{ }^{*} \operatorname{begin}(i)={ }^{*} \operatorname{begin}(i+j)$

(PBE1') $\forall p \exists i, j \cdot p={ }^{*} \operatorname{begin}(i) \& p={ }^{*} e n d(j)$

$\left(\mathrm{PBE} 2{ }^{\prime}\right) \quad \forall i, j \cdot i \cdot j \equiv\left({ }^{*} e n d(i)={ }^{*} \operatorname{begin}(j)\right)$

(PBE3') $\forall p, q . p<q \equiv \exists i \cdot p={ }^{*} \operatorname{begin}(i) \& q={ }^{*} e n d(i)$

\section{Moments}

Some points in time seem to be where intervals meet, but others also seem to have enough substance that events can happen in them. For example, a light bulb burns out with a flash, plunging the room into darkness, and in that instant one sees a face at the window, caught in the intensity of the flash. Or a bat hits a ball, and there is a loud "crack" and the bat breaks. Other examples can be given. All of these seem like real events which occupy points rather than intervals. We will introduce the idea of a very short interval, called a moment, to be the times which such events occupy.

A moment is an interval with no internal structure. It therefore does not overlap any other interval, and contains no other intervals (Theorem MO1). One can sum it up by saying that there are no points inside it. Formally we can define: 
$\forall i . T R U E-I N T E R V A L(i) \equiv \exists a, b, c, d . a: i: d \& a: b: c: d$

$\forall i . M O M E N T(I) \equiv \neg T R U E-I N T E R V A L(i)$

Thus, a true-interval has at least two sub-intervals (which might in turn be moments or true-intervals)--one that STARTS it and one that FINISHES it. Another way of stating the definition of a true-interval would be that it is the result of some union operation, i.e.,

$$
\forall i . T R U E-I N T E R V A L(i) \equiv \exists a, b . i=a+b
$$

Before we continue, it is important to remember that all of the earlier theorems were proven before any distinction was made between moments and true-intervals, so they all hold for both classes; none of the proofs ever depended on the decomposability of an interval. These definitions allow us to prove that two moments cannot overlap in any sense of the term, yet they can meet each other. More precisely,

$$
\forall i, j . \operatorname{MOMENT}(i) \& \operatorname{MOMENT}(j) \supset i(<m=m i>) j
$$

Moments are very simple intervals. While a moment may be a time of a change, nothing changes within that time (i.e., there are no subperiods). In a reasonably complicated dynamic world, probably the only way to achieve this is for a moment to be of very short duration. We say that a flash "freezes" time, for example: there can be no change, and hence no movement or event, during an interval that short. We might also take clock ticks to be moments. However, there is nothing in the formal theory that talks of the durations of moments, and no reason why they must be thought of as short, or as all having the same duration. Clearly, moments are different from points. They belong in different categories: things can be true or false during a moment, which is meaningless for points. But in many respects moments are pointlike entities. For example, they can be identified with the ends of the intervals they meet, since we have the following:

(MO3) $\forall i, j, k .(\operatorname{moment}(i) \& \operatorname{moment}(j) \& k: i \& k: j) \supset i=j$

Proof: Suppose not; then end $(i) \neq e n d(j)$, hence by M2 there is some $k$ with either end $(i) ; k$;end $(j)$, or symmetrically. But then $j=i+k$, so moment $(j)$ is false. Similarly in the other case. QED.

A similar lemma holds for the case where $i: k$ and $j: k$. We cannot define functions begin-moment and end-moment, and other obvious variations such as last-moment and first-moment, since there is no guarantee that these moments in fact exist. It may be that there are no moments, that all intervals have subintervals, so that the set of points is a dense linear order. We will discuss 


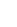



this in the next section. But if they exist, they are unique. We can in any case define relations such as just-after:

$$
\forall i, j . j u s t-\operatorname{after}(i, j) \equiv(i \cdot j \& \operatorname{moment}(j))
$$

and just-before similarly, and last-moment:

$$
\forall i, j . \text { last-moment }(i, j) \equiv \exists k . i: k \& j: k \& \operatorname{moment}(j)
$$

and first-moment similarly. In the dense model, these are everywhere false, but when they are true we can derive point-like properties, for example:

$$
\begin{aligned}
& \forall i, j .(\exists m, n \text {. first-moment }(i, m) \& \text { first-moment }(j, m) \\
& \text { \& last-moment }(i, n) \text { \& last-moment }(j, n)) \supset i=j
\end{aligned}
$$

and a similar lemma holds with just-before and just-after. Thus moments can be thought of as sufficient to locate the endpoints of intervals, and each interval determines a pair of "end-moments."

It seems promising to try to use moments as points, to make the similarity more compelling. However, it is easy to show that if just-before $(i, m)$ and firstmoment $(i, n)$, then $m: n$, and so $m \neq n$, so that these two kinds of end-moment must be distinct from one another, so we do not have the uniqueness which characterizes points. And if we decide to choose one and stick to it, then difficulties arise which are reminiscent of those discussed in the introduction. For example, suppose we decide to use the just-after/just-before vocabulary as a way of treating moments as points. We would want the "beginning" of an interval $j$ to be identical with the "end" of $i$ just when $i$ meets $j$, but this immediately fails, since if $i: j$ then $j u s t-a f t e r(i, m)$ is true exactly when firstmoment $(j, m)$ is, so we are obliged to use the other way of talking about the next interval. This is reminiscent of the problems over open and closed intervals with which we began, since this distinction is precisely between the "ends" of an interval which are included in it and those which are not.

The most obvious structural difference between points and moments is that moments can meet other intervals, and hence stand between them. Thus if $i ; p ; j$, then $i$ and $j$ meet: there is nothing between them. But if $i: m: j$ for a moment $m$, then it follows immediately that $i$ does not meet $j$. It is interesting to see what happens if we try to ignore this. Suppose we define a relation ALMOST-MEETS, written as an infix double colon, to mean that two intervals are separated, if at all, by only a moment. Consider the axiom M1, with ALMOST-MEETs instead of MEETS, and suppose that $i:: j, i:: k$, and $l:: j$. We might have $i: j, i: m: k$, and $l: n: j$, so that $l: n: m: k$. Now $l$ and $k$ are separated by the sum of two moments, which is itself of course more than a moment. The "small errors" can add up, as it were, and produce larger errors. This sort of exercise emphasizes the essential difference between points and intervals, even the smallest possible intervals. 
To emphasize this furthur, while moments have no points inside them, they do have endpoints, and these are different: the beginning and end of a moment are distinct, just as they are for other intervals. This is one of the less intuitive aspects of our theory, for it distinguishes between, for example, the beginning and the end of a flash of lightning. A fully comprehensive account of this aspect of temporal intuition would probably involve thinking about changes of scale, so that a moment at one scale would "shrink" to a point at a coarser scale. We have not gone into this in detail, however.

\section{Discussion}

It is interesting to interpret these axioms in various possible models. The simplest one is discrete time: intervals are pairs of integers $\langle n, m\rangle$ with $n<$ $m$, and $\langle n, m\rangle:\langle m, k\rangle$. Then a moment is a nondecomposable interval $<n, n+1\rangle$, and nests pick out integers, the places "between" moments. In this model there is a clear distinction between moments and points. We can also define several models based on the real line. For example, time intervals can be mapped into open or closed real intervals: however, then times can never MEET. A simpler continuous model, based on the integer model above, defines time intervals as pairs of reals $\langle a, b\rangle$, with $\langle a, b\rangle:\langle b, c\rangle$. Following through the axiomatic definitions with this as a basis makes nests define points on the real line, as expected, but now there are no moments at all, since even the smallest interval is decomposable. We might try to extend the model to allow intervals of the form $\langle a, a\rangle$, which would qualify as moments, but now consider $\langle a, b\rangle,\langle b, b\rangle$ and $\langle b, c\rangle$. By our definitions, the first MEETs the last, yet they have the second between them, so the first is BEFORE the last, violating the ordering axiom. We have tried to fit real, substantial--though very small-time intervals into merely mathematical "places," and they don't fit.

However, another possible model is one which mixes these, using the same definitions of interval and MEET (from which all else follows) but allowing parts of the time line to be discrete and parts to be continuous. Intuitively, if we have only coarse time measuring tools available, then we can treat time as discrete, but the possibility always remains of turning up the temporal magnification arbitrarily far, if we have access to events which can make the finer distinctions, distinctions which can split "moments" into smaller and smaller parts.

Our axiomatic theory allows all of these models and others; it is uncommitted as to continuity or discreteness of the sequence of times, yet is powerful enough to support a great deal of the temporal reasoning of common sense.

\section{Acknowledgements}

We are grateful to Dana Scott for bringing Hamblin's work to our attention and emphasizing some of the subtleties in constructing models of points. We also wish to thank Henry Kautz for pointing out the power of axiom M2, namely 
the lemma ML1. Hans Koomen helped with an initial formulation of moments as "endpoints," which subsequently developed into the separate formulations of points and moments as discussed in this paper. Thanks also to Peter Ladkin for many interesting discussions related to the topics in this paper, and to Jay Weber for his comments on the final draft. 


\section{Appendix: Proofs}

The proofs in this section follow the development of the paper, and the numbering between the two is consistent. The proofs are presented here with only a minimum of motivation and assume the paper is used for this purpose.

\section{The Properties of Intersection}

Def'n: $I ! J$ is the interval that exists if there exists a subinterval common to both $I$ and $J$, i.e.,

$$
\exists i .(i I N I) \&(i I N J) \supset(I ! J I N I) \&(I ! J I N J)
$$

and it is the largest common subinterval, i.e.,

$$
\forall i \text {. ( i IN I) \& ( } i I N J) \supset i I N I ! J
$$

Lemma IL1: $I ! J$ is unique if it exists.

Proof: Assume $A$ and $B$ are both intersections of $I$ and $J$, then by axiom I1 above, $A I N I$ and $A I N J$, then by axiom I2 above we can conclude that $A I N B$. Using the same argument, we can show that $B I N A$, thus $A$ must be equal to $B$.

Lemma IL2: If two intervals overlap in any way, then their intersection exists. The intersection is constructed from the definitions of each of the possible relationships between two intervals $I$ and $J$.

Proof that intersection exists in various cases:

Case 1: Assume that $I I N J$, i.e.,

\section{STARTS J, I FINISHES J, I DURING $J$ or I EQUAL $J$}

then we can see that $I ! J=I$ by showing that I1 and I2 hold: Since $I I N I$ by definition, we have $I I N I$ and $I I N J$ satifying I1. But since $I ! J$ must be in $I$, any $i I N I ! J$ is in $I$, thus $I$ satisfies I2.

Case 2: Assume IOVERLAPS J, then $\exists a, b, c, d, e$<smiles></smiles>

We will now prove that $I ! J=c$, by showing that it satisfies I1 and I2:

1) Since $c I N I$ and $c I N J$, axiom I1 is trivially satisfied by $c=I ! J$ 
2) Assume i $I N I$ and $i I N J$, for any $i$, then

$i$ IN I \& I FINISHED-BY $c \supset i(b$ moffis $d) c$

iIN $J$ \& $c$ STARTS $J \supset i(s$ si $=$ mi oi $d$ f $) c$

Intersecting these constraints, we have $i(s=f d) c$, which implies by definition of IN that $i$ IN $c$ and hence I2 holds.

So, every subinterval of $I ! J$ is in $c$, thus $c=I ! J$.

Case 3: If $I: J$ or I BEFORE $J$, then there is no intersection.

This is a trivial proof by contradiction. QED

Lemma IL3: A) If $i=a+b$ and $j: b$, then $i ! j$ exists and is equal to $a ! j$

B) If $i=a+b$ and $a: j$, then $i ! j$ exists and is equal to $b ! j$.

Proof (of A; B is similar): We show that $i ! j$ exists, as follows. By M3, we know there are a $k$ and $l$ such that $k: i$ and $l: j$. Applying M2, there are three possibilities:

Case 1: $\quad k: j$, in which case, given $k: a$ (since $j=a+b$ ) we have $j=a$, hence $i=$ $j+b$. It is easily shown that $j$ satisfies the definition of $i ! j$.

Case 2: $\exists m \cdot k: m: j$, which means that $a=m+j$ (since $k: a: b$ and $k: m: j: b$ ), hence $i=m+j+b$. Again, $i ! j=j$.

Case 3: $\exists m . l: m: i$, which means that $j=m+a$ and $i=a+b$. It can be easily shown that $a$ satisfies the definition of $i ! j$.

So $i ! j$ exists. To show it is equal to $a ! j$, consider $a ! j$ in each of the three cases:

Case 1: $a=j$, so $a ! j=j$

Case 2: $\quad a=m+j$, so $a ! j=j$

Case 3: $j=m+a$, so $a ! j=a$

Hence $i ! j=a ! j$ in all cases. QED.

\section{Properties of the Temporal Reasoner}

The proof of the transitivity relationships is tedious and will not be presented in full here. Rather we present a representation sample of the proofs, which all follow the same pattern.

Consider deriving the transitivity table for BEFORE from first principles: 
Assume $A$ BEFORE $B$, i.e., $\exists i$ such that $A: i: B$

1) If $B B E F O R E C$, then $\exists j . B: j: C$, thus $A$ BEFORE $C$ since $A:(i+B+j): C$;

2) If $B: C$, then $A$ BEFORE $C$ since $A:(i+B): C$;

3) If $B$ OVERLAPS $C$, then $\exists j, k . j: B \& j: k: C$ thus $A$ BEFORE $C$ since $A:(i+k): C$;

4) If $B$ STARTS $C$, then $\exists j \cdot j: B \& j: C$, hence by axiom M1, $i: C$, thus $A: i: c$ and thus $A B E F R E C$

5) If $B$ FINISHES $C$, then $\exists j, k, l . j: C \& j: k: B \& B: l \& C: l$

Applying axiom M3 to $A: i$ and $j: C$, we get either

(1) $A: C$, or

(2) $\exists m . A: m: C$ (i.e., (2) $A B E F O R E C$ ) or

(3) $\exists n \cdot j: n: i$

Looking at this last case we have:

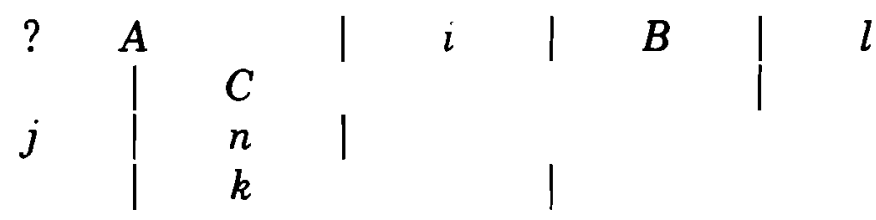

The only uncertainty now concerns the beginning of $A$. We know by axiom M4 that there exists an $o$ such that $o: A$. Using axiom M3 to combine this with the fact that $j: C$ we get either

$o: C$, (hence $A$ STARTS $C$ ), or

$\exists m$.o:m:C (hence A OVERLAPS C), or

$\exists n \cdot j: n: A$ (hence $A$ DURING C)

Hence, by applying M3 repeatedly on the definitions of the interval relationships, we have derived that

$A(: b s \circ d) C$

6) If $B D U R I N G C$, we get essentially the same proof as in the above case, deriving $A(: b s \circ d) C$.

7) If $B E Q U A L S C$, then we prove $A B E F O R E C$ by a simple application of axiom M1. 
8) If $B A F T E R C$, then there exists $j . C: j: B$.

We end up applying axiom M3 six times in this proof, each time except the last produces two possible relationships between $A$ and $C$, and a third possibility that is further developed. The last application produces the final three possible relationships that could hold between $A$ and $C$. Thus, these two facts produce no information about the relationship between $A$ and $C$.

9) If $B M E T-B Y C$, (i.e., $C: B$ ).

We know from axiom M5 that there exists a $j . j: C$. Using axiom M3 on A:i and $j: C$ we get either

(1) $A: C$, or

(2) $\exists m$.A:m:C (i.e. (2) $A B E F O R E C$ ), or

(3) $\exists n . j: n: i$, i.e.,<smiles>[VH]I</smiles>

We know by axiom M5 that there exists a $k$ such that $k: A$. Combining $k: A$ and $j: C$ using axiom M3 we get either

$k: C$ (hence A STARTS C), or

$\exists m . k: m: C$ (hence A OVERLAPS C), or

$\exists n . j: n: A$ (hence $A D U R I N G C$ ).

Thus we have

$A(m b s \circ d) C$

10) If $B O V E R L A P P E D-B Y C$, we get the same results as in (9), by the same proof, thus $A$ ( $m b s o d) C$.

11) If $B$ STARTED-BY $C$, then $\exists j . j: B \& j: C$

Now by axiom M1, we have $i: C$, thus $A B E F O R E C$.

12) If $B$ FINISHED-BY $C$, then $\exists j, k . j: B \& j: k: C$

thus we have $A:(i+k): C$, hence $A B E F O R E C$

13) If $B$ CONTAINS $C$, then we have the same proof as in (12).

This completes the entries for the transitivity table for the BEGIN relation. The proofs for the remaining entries introduce no new complexities. 


\section{Time Points}

The proofs of P1 through P5 and Lemma PL2 are given here, assuming axioms PBE1-PBE3 and M1-M5.

Theorem P1: $\forall p . \neg(p<p)$

Proof: By PBE3, $p<p \equiv \exists i . p=$ begin(i) \& $p=$ end(i). But then by PBE2, this entails $i: i$, contradicting Lemma ML1. QED.

Theorem P2: $\forall p, q .(p<q) \supset \neg(q<p)$

Proof: Assume $p<q$, then by PBE3, $\exists i . p=\operatorname{begin}(i) \& q=$ end(i). Assume $q$ $<p$, then by PBE3, $\exists j . q=\operatorname{begin}(j) \& p=\operatorname{end}(j)$. Thus begin $(i)=p=$ end $(j)$ and $\operatorname{end}(i)=q=$ begin $(j)$; thus $i: j$ and $j: i$, contradicting Lemma ML2. QED.

Theorem P3: $\forall p, q, r .(p<q) \&(p<r) \supset(p<r)$

Proof is in main paper.

Theorem P4: $\forall p, q .(p<q) \oplus(q<p) \oplus(p=q)$

Proof: Given $p, q$, by PBE1 we know $\exists i, j \cdot p=e n d(i)$ and $q=\operatorname{begin}(j)$. By axiom M2, exactly one of the following holds:

Case 1: $\quad i: j$, then by PBE2, end $(i)=\operatorname{begin}(j)$; thus $p=q$

Case 2: $\exists m$. $i: m: j$, then by PBE2, $p=e n d(i)=\operatorname{begin}(m)$, and $q=\operatorname{begin}(j)=$ end $(m)$. Thus by definition PBE3, $p<q$.

Case 3: As in Case 2, but $q<p$. QED.

Lemma PL2: $\forall i$. begin(i)<end(i).

Proof: We show this by disproving the other two cases in Theorem P4. Assume begin $(i)=$ end $(i)$; then $i: i$, contradicting ML1. Assume end $(i)<$ begin $(i)$; then by PBE3, $\exists m$. begin $(m)=e n d(i)$ and $e n d(m)=\operatorname{begin}(i)$. Thus $m: i$ and $i: m$, contradicting ML2. Using P4, we conclude begin( $i)<$ end(i). QED.

Theorem P5: $\forall p . \exists q, r . q<p<r$

Proof by Construction: Using PBE $1, \exists i, j \cdot p=\operatorname{begin}(i)$ and $p=e n d(j)$. Let $q=$ begin $(j)$ and $r=e n d(i)$. Using Lemma PL2, we can show $q<p$ and $p<r$. QED.

Lemma PL3: $\forall i, j, p, q .(p ; i ; q \& p ; j ; q) \supset i=j$

Proof: Translating the notation, we have $p=\operatorname{begin}(i) \&$ end $(i)=q \& p=$ $\operatorname{begin}(j)$ and end $(j)=q$. Using PBE1, there exists an interval $k$ such that end $(k)$ 
$=p=\operatorname{begin}(i)=\operatorname{begin}(j)$. Hence $k: i$ and $k: j$. Similarly, there is an interval $l$ such that $i: l$ and $j: l$. hence by M5, $i=j$. QED.

Lemma PL4: $\forall i, j . i: j \supset(\forall p . i ; p \equiv p ; j)$

Proof: Assume $i: j$, thus end $(i)=\operatorname{begin}(j)$; thus end $(i)=p$. Then $p$ must equal $\operatorname{begin}(j)$, and vice versa. QED.

Lemma PL5: $\forall i . \exists p, q \cdot p ; i ; q$

Proof: Trivial, as $p=\operatorname{begin}(i)$ and $q=e n d(i)$. QED.

IV. Proofs About Nests

Defn: ${ }^{*} \operatorname{begin}(I)=\{p \mid \exists b, c . \quad b: I \& b: c \&$ $p=b+c \operatorname{OR} p=c \operatorname{OR} p=b\}$, i.e.,

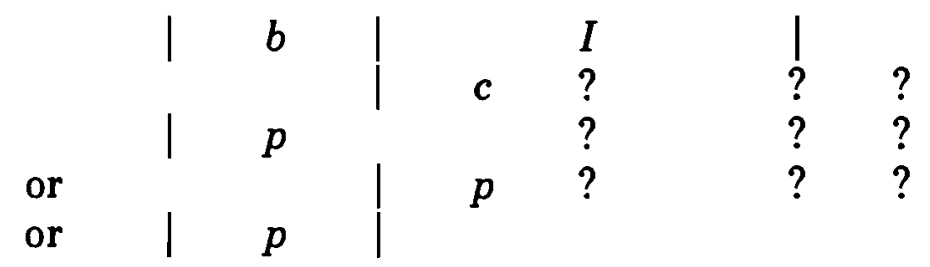

Def'n: $\quad *$ end $(I)=\{p \mid \exists a, b . I: b \& a: b \&$ $p=a+b \operatorname{OR} p=a \mathrm{OR} p=b\}$, i.e.,

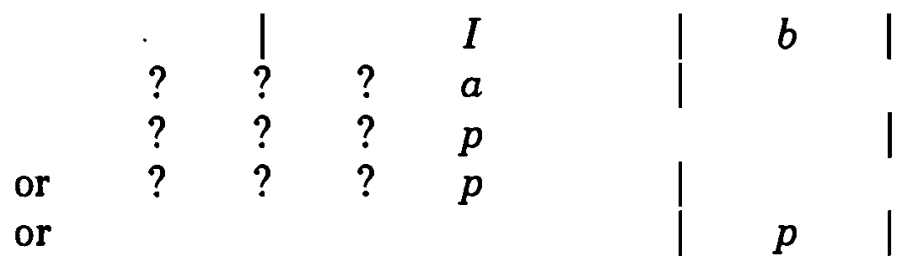

These sets are always non-null. These could also be defined by the following:

*begin $(I)=\{p \mid p$ (o s $m$ fidie si) $I\}$

${ }^{*}$ end $(I)=\{p \mid p$ (oif mifidie si) $I\}$

For convenience, we can define a nest as a beginning or an ending:

$$
N E S T(p) \equiv \exists I \cdot p=B E G I N(I) \text { OR } p=E N D(I)
$$

Def'n: for any two NESTS, $N$ and $M$

$$
N<M \equiv \exists n, m . n \text { is in } N \& m \text { is in } M \& n<m
$$

Lemma NL1: $I$ is in *begin( $(I)$ and *end(I) 
Proof: This is trivial, because $I$ satisfies the definition of both. That is, if we let $j$ $=c=I$ in the definition of ${ }^{*} \operatorname{begin}(i)$, we get $\exists b . b: I \& b: I \&(j=b+I \vee I=I \vee j$ $=b)$. This is trivially true. The proof for *end $(I)$ is similar. QED.

Lemma NL2: If $i$ and $j$ are in a nest $N$, then either $i: j, j: i$, or $i ! j$ exists and is in $N$.

Proof: Let $N={ }^{*}$ begin( $(I)$ (the proof for $N={ }^{*} e n d(I)$ is similar) and assume that $i$ and $j$ are in *begin(I). We know there are $a, b$ such that $a: I, a: b$, and either $i=$ $a+b, i=a$, or $i=b$. We know there are $c, d$ such that $c: I, c: d$, and either $j=$ $c+d, j=c$, or $j=d$. Given all the above, it is simple to show using M1 that $c: b$ (since $a: I, c: I$, and $a: b$ ) and $a: d$ (since $a: I, c: I$, and $c: d$ ). We can now examine each case one at a time, showing that either $i ! j$ exists, or $i: j$, or $j: i$. The results are shown in Table 1. For example, if $i=a$ and $j=c+d$, then since we know $a: d$ (hence $i: d$ ), Lemma IL3 shows that $i ! j$ exists and equals $c ! i$ (which is $c ! a$ ).

\begin{tabular}{|c|c|c|c|}
\hline$j$ & $a+b$ & $a$ & $b$ \\
\hline$c+d$ & $?$ & $i ! j=c ! a$ & $i ! j=b ! d$ \\
\hline$c$ & $i ! j=c ! a$ & $i ! j=c ! a$ & $j: i$ \\
\hline$d$ & $i ! j=b ! d$ & $j: i$ & $i ! j=b ! d$ \\
\hline
\end{tabular}

Table 1

To prove the lemma, we now only need (1) to show that $a ! c$ and $b ! d$ are in * begin(I) when they exist, and (2) to deal with the one case where $i=a+b$ and $j$ $=c+d$.

Part 1: Consider $a ! c$. We know $a: I$ and $c: I$, and since $a ! c$ is the largest interval in both $a$ and $c$, it is not hard to show that $(a ! c): I$. Since both meet $I$, it is either the case that there is an $x$ such that $a=x+c$, and then $a ! c=c$, which meets $I$, or there is an $x$ such that $c=x+a$, and then $a ! c=a$, which meets $I$. Thus $a ! c \epsilon$ *begin $(I)$ by definition. Similarly $b ! d \epsilon{ }^{*}$ begin $(I)$ by definition.

Part 2: The last case is where $i=a+b$ and $j=c+d$. To summarize, we know $a: I, a: b, c: I, c: d, c: b$, and $a: d$. By M3, we know that there exists $f, g$ such that $f: i$, hence $f: a$ and $g: b$, and hence $g: c$. Using M3 we get three posibilities:

$f: c$, in which case $a=c$

$\exists m . f: m: c$, in which case $a=m+c$

$\exists n \cdot g: n: a$, in which case $c=g+a$ 
Similarly, there are intervals $h$ and $l$ such that either

$$
\begin{aligned}
& b=d \\
& b=d+h \\
& d=b+l
\end{aligned}
$$

Considering each of these possibilities, we find the values for $i ! j$ shown in Table 2. In each case, it is easy to show that $i ! j$ is in *begin(I). QED.

$$
\begin{array}{r|c|c|c|}
\multicolumn{1}{c}{} & a=c & a=m+c & c=g+a \\
b=d & i ! j=i=j & i ! j=c+d & i ! j=a+d \\
\cline { 2 - 4 } b=d+h & i ! j=a+d & i ! j=c+d & i ! j=a+d \\
\cline { 2 - 4 } d=b+l & i ! j=a+b & i ! j=c+b & i ! j=a+b \\
\cline { 2 - 4 } & &
\end{array}
$$

Table 2

Theorem N1: For any two nests $N$ and $M$ : then either $N<M, M<N$ or $N=$ $M$.

Proof: Assume $N<M$, then by definition there exists an $n$ in $N$ and an $m$ in $M$ such that $n<m$. By definition we know that $N$ is not equal to $M$. We can show that $N$ is not greater than $M$ by contradiction: assume there exists an $a$ in $N$ and $b$ in $M$ such that $a>b$, then we know by Lemma NL2 that

either $a: n, n: a$, or $a ! n$ exists and is in $N$, and

either $b: m, m: b$, or $b ! m$ exists and is in $M$. Now

IF $b ! m$ exists, then

$b ! m I N b \& b ! m I N m \& n<m \& b<a$

$\supset n<b ! m<a$, but if this is the case then $a ! n$ does not exist and $a$ does not MEET $n$. A contradiction

So we are left with the possibility that $b: m$ or $m: b$, and $a: n$ or $n: a$. But given $a>$ $b$ and $n<m$, each possible pairing of relations is inconsistent. QED.

Thus we have shown that $\langle$,$\rangle , and =$ are mutually exclusive. To show that one must hold between any two nests we show that if it is not the case that $N<$ $M$ or $M<N$, then $M=N$.

Assume that $N$ is not less than $M$ and vice versa. Then pick an arbitrary $n$ in $N$. We will prove that $n$ must be in $M$. 
Case 1: Assume $M=B E G I N(I)$

then there is an $m^{\prime}$ in $M$ such that $m^{\prime}: I$.

Furthermore, by the hypothesis we know that $n$

cannot be $<$ or $>m^{\prime}$, i.e.,

$n$ (o oiddi m missiffie) $m^{\prime}$

By applying the transitivity axioms, it is simple to show that $n(<$ o do fi $s$ si $=$ $m$ ) $I$. If $n<I$, then $N<M$, a contradiction. Hence $n$ (o di fi s $s i=m$ ) $I$, which means, by definition, that $n$ is in $B E G I N(I)$.

Case 2: Assume $M=E N D(I)$. The same proof goes through using $m$ ' such that $I: n$.

We will prove PBE1 through PBE2 for the nest constructs *begin and *end. With this, all the previous theorems about points will automatically be valid for nests. PBE2 will be proven first, as it is useful for showing PBE1.

(PBE2) $\quad \forall i, j, i: j \equiv *$ end $(i)={ }^{*} \operatorname{begin}(j)$

Proof: Assume I:J. Then we can show any interval $k \epsilon^{*}$ end(I) is also in ${ }^{*} \operatorname{begin}(J)$ and vice versa. Assume $k \epsilon^{*}$ end $(I)$. Then by definition there exists $a, b$ such that $I: b$ and $a: b$. By M1, $a: J$ since $I: J$. We know that either $k=a+b, k$ $=a$, or $k=b$.

Case 1: $\quad k=a+b$; then $k \epsilon^{*}$ begin $(J)$ by definition (since $a: J$ and $k=a+b$ ).

Case 2: $\quad k=a$; then $k \epsilon^{*} \operatorname{begin}(J)$ since $a: J$ and $k=a$.

Case 3: $\quad k=b$; then $k \epsilon^{*}$ begin $(J)$ since $a: J, a: b$, and $k=b$.

The other direction is similar (i.e., if $k \epsilon^{*} \operatorname{begin}(I)$ then $k \epsilon^{*}$ end $(I)$ ).

For the other direction of the biconditional, assume *end $(i)={ }^{*}$ begin $(j)$. Let $i: k$, thus $k \epsilon^{*}$ end $(i)$. Now $k \epsilon^{*}$ begin $(j)$, since ${ }^{*} \operatorname{begin}(j)={ }^{*}$ end $(i)$. Thus there are intervals $b, c$ such that $b: j$ and $b: c$, and either $k=b+c, k=b$, or $k=c$. Consider the cases $k=b+c$ or $k=b: i: k \Rightarrow i: b$ and $b: j \Rightarrow i$ BEFORE $j$. But then $i \epsilon$ ${ }^{*}$ end $(i)$ and $j \epsilon * \operatorname{begin}(j)$ implies *end $(i)<* \operatorname{begin}(j)$, a contradiction of Theorem $\mathrm{N} 1$. Thus it must be the case that $k=c$. Hence $i: k, b: j, b: k$ imply by M1 that $i: j$. QED.

(PBE1) $\forall p \exists i, j \cdot p={ }^{*} \operatorname{begin}(i) \& p={ }^{*} e n d(j)$

Proof: Nests are defined to be either of form *begin $(I)$ for some $I$, or *end $(J)$ for some $J$. All that is left to show is that for each nest of form *begin(I), there is a $J$ 
such that ${ }^{*}$ begin $(I)={ }^{*}$ end $(J)$. But M1 says such a $J$ exists. For any $I$, there is a $J$ such that $I: J$, and the proof of PBE2 above shows *begin $(I)={ }^{*}$ end $(J)$.

Lemma PL1: $\forall i, j . i: j \supset^{*} e n d(j)={ }^{*}$ end $(i+j)$

$$
\forall i, j . i: j \supset{ }^{*} \operatorname{begin}(i)={ }^{*} \operatorname{begin}(i+j)
$$

Proof: This follows from PBE2 just as in the text. If $i: j$, then there is a $k$ such that $j: k$ and $(i+j): k$. Hence ${ }^{*}$ end $(j)={ }^{*} \operatorname{begin}(k)={ }^{*}$ end $(i+j)$. The other direction is similar.

(PBE3) $\quad \forall p, q \cdot p<q \equiv \exists i \cdot p={ }^{*} \operatorname{begin}(i) \& q={ }^{*} e n d(i)$

Proof: One direction is trivial. Assume $\exists i ., p={ }^{*}$ begin( $i$, and $q={ }^{*}$ end( $i$ ). Then we know by M3 that $\exists j, k$ such that $j: i: k$. Furthermore, $j \in{ }^{*}$ begin( $i$ ) and $\mathrm{k}$ $\epsilon{ }^{*}$ end $(i)$ by definition. Since $j$ BEFORE $k, p<q$ by definition. The other direction is as follows. Assume $p<q$. Then by definition there are two intervals $n$ and $m$ such that $n \in p$ and $m \in q$ and $n$ BEFORE $m$.

By PBE1, there are intervals $I$ and $J$ such that $p={ }^{*}$ begin $(I)$ and $q={ }^{*}$ end $(J)$. Furthermore, the definition of BEFORE gives an interval $a$ such that $n: a: m$. We can construct an interval $k$ with the desired properties by considering the six possible ways $m$ and $n$ might relate to $I$ and $J$. By definition, there are intervals $b, c$ such that $b: I$ and $b: c$ where $n=b+c$ or $n=b$ or $n=c$. Likewise, there are intervals $d$,e such that $J: e$ and $d: e$ where $m=d+e$ or $m=d$ or $m=e$.

Case 1: Let $n=b+c$ or $n=c$. Let $m=d+e$ or $m=d$. Remember $n: a: m$. Let $k=c+a+d$. We know $k$ is well-formed since $n: a$ implies $c: a$ and $a: m$ implies a:d. By Lemma PL1, PBE2 using b:c and b:I, *begin $(k)=$ ${ }^{*} \operatorname{begin}(c)={ }^{*}$ end $(b)={ }^{*}$ begin $(I)=p$. Similarly, ${ }^{*}$ end $(k)={ }^{*}$ end $(d)=$ ${ }^{*} \operatorname{begin}(e)={ }^{*} e n d(J)=q$. Thus $k$ is the desired interval.

Case 2: Let $n=b, m=e$. Then $k=a$ is the solution: *begin $(k)=* \operatorname{begin}(a)=$ ${ }^{*}$ end $(n)={ }^{*}$ end $(b)={ }^{*} \operatorname{begin}(I)=p$; and ${ }^{*}$ end $(k)={ }^{*}$ end $(a)=$ ${ }^{*} \operatorname{begin}(m)={ }^{*} \operatorname{begin}(e)=*$ end $(J)=q$.

Case 3: Let $n=b, m=d+e$ or $m=d$. Then $k=a+d$ is the solution.

Case 4: Let $m=e, n=b+c$ or $n=c$. Then $k=c+a$ is the solution.

\section{Discrete Time and Time Points}

Def"n: TRUE-INTERVAL(I) $\equiv \exists a, b, c, d \cdot a: I: d \& a: b: c: d$

Def'n: $M O M E N T(I) \equiv \neg T R U E-I N T E R V A L(I)$

As a consequence of these definitions, we see that for every true interval $I$, there is an interval $S$ that STARTS I, and an interval $F$ that FINISHES I. Except in the case where $S$ and $F$ are both moments, we can also see that there will exist 
an interval $D$ that is DURING $I$ as well. We can prove that moments have no subintervals, which is equivelent to saying that nothing ever starts, finishes, is during, overlaps or is overlapped by a moment.

Theorem MO1: MOMENT(i) $\supset \neg \exists j . j$ (ooi starts finishes during) $i$

Proof: By contradiction, each one of these relations contradicts the exclusive-or in (M3) above. For example: assume $J$ overlaps $D$ \& $M O M E N T(D)$. By definition of overlaps: $\exists a, b, c, d, e$ such that

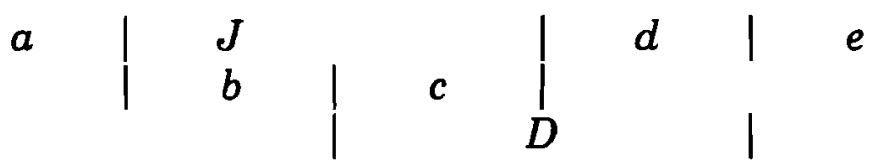

mapping $b \rightarrow j, c \rightarrow k, d \rightarrow l, e \rightarrow m$, we satisfy two disjuncts in axiom (M3), a contradiction.

Theorem MO2: For all $I, J . M O M E N T(I) \& M O M E N T(J) \supset$

$$
I(<m=m i>) J
$$

Proof: Using Lemma 4-1 we have that $I(<m=m i>$ si fi $d i$ ) $J$ (by taking all the alternatives not eliminated by Lemma 4-1). Similarly, we have $J(<m=$ $m i>$ si fi di) $I$. Combining these two, we get $I(<m=m i>) J$, as desired. 


\section{References}

Allen, J.F., "Maintaining knowledge about temporal intervals," TR 86, Computer Science Dept., U. Rochester, January 1981; Communications of the ACM 26, 11, 832-843, November 1983.

Allen, J.F., "Towards a general model of action and time," Artificial Intelligence 23, 2, July 1984 .

Hamblin, C.L., "Instants and intervals," in J.T. Fraser, F.C. Haber, and G.H. Muller (Eds). The Study of Time. New York: Springer-Verlag, 1972.

Kamp, J.A.W., "Instants and temporal reference," in A. Von Stechow (Ed). Semantics from Different Points of View. Springer-Verlag, 1979.

Mourelatos, A.P.D., "Events, processes, and states," Linguistics and Philosophy 2, 415-434, 1978.

Van Benthem, J.F.A.K. The Logic of Time. Reidel, 1982. 